\title{
There are no Barker arrays having more than two dimensions
}

\author{
Jonathan Jedwab Matthew G. Parker
}

5 June 2006 (revised 7 December 2006)

\begin{abstract}
Davis, Jedwab and Smith recently proved that there are no 2-dimensional Barker arrays except of size $2 \times 2$. We show that the existence of a $(d+1)$-dimensional Barker array implies the existence of a $d$-dimensional Barker array with the same number of \pm 1 elements. We deduce that there are no Barker arrays having more than two dimensions, as conjectured by Dymond in 1992.
\end{abstract}

\section{Introduction}

We define a length $s$ binary sequence to be a one-dimensional array $A=(a[i])$ whose elements satisfy

$$
a[i]= \begin{cases}-1 \text { or } 1 & \text { if } 0 \leq i<s \\ 0 & \text { otherwise }\end{cases}
$$

The aperiodic autocorrelation function of a length $s$ binary sequence $A=(a[i])$ is given by

$$
C_{A}(u):=\sum_{i} a[i] a[i+u] \text { for integer } u
$$

and measures the extent to which a binary sequence resembles a shifted copy of itself.

Since the 1950s, digital communications engineers have sought to identify binary sequences for which the absolute values of the aperiodic autocorrelation function are collectively small, for application in synchronisation, pulse compression and especially radar [11]. From this point of view an ideal length $s$ binary sequence $A$, known as a Barker sequence, is one for which

$$
\left|C_{A}(u)\right| \leq 1 \text { for all } u \neq 0 .
$$

For example, $[+++++--++-+-+]$ is a length 13 Barker sequence (where + and - represent sequence elements 1 and -1 respectively). For any length $s$ binary sequence $A$ and integer $u$ satisfying $|u|<s$, the autocorrelation $C_{A}(u)$ is the sum of exactly $s-|u|$ terms, each of which is \pm 1 , and so $C_{A}(u) \equiv s+u \quad(\bmod 2)$. Therefore no

J. Jedwab is with Department of Mathematics, Simon Fraser University, 8888 University Drive, Burnaby BC, Canada V5A 1S6. He is grateful for support from NSERC of Canada.

M.G. Parker is with Department of Informatics, High Technology Center in Bergen, University of Bergen, Bergen 5020, Norway. He is grateful to Simon Fraser University for hospitality during 2006. 
binary sequence $A$ can have a smaller value of $\left|C_{A}(u)\right|$ than a Barker sequence, for any $u$. However the only non-trivial lengths $s$ for which Barker sequences are known to exist are $2,3,4,5,7,11$ and 13, and it has been conjectured since at least 1963 [9] that no other sequence lengths are possible (see [4] for historical background):

Conjecture 1.1. There is no Barker sequence of length $s>13$.

Conjecture 1.1 is known to hold for odd $s$ :

Theorem 1.2 (Turyn and Storer $1961[8]$ ). There is no Barker sequence of odd length $s>13$.

Turyn's classical paper [10] of 1965 established that Conjecture 1.1 is true for (even) $s<12,100$, by exploiting a connection with cyclic Hadamard difference sets. In 2005 Leung and Schmidt [6] improved this to $s \leq 10^{22}$ using the "field descent method" (see [7] for a thorough description of this ground-breaking method).

Once it became apparent that the ideal behaviour given by a Barker sequence is unlikely to be achieved beyond length 13, researchers explored two relaxations of the Barker condition. One relaxation is to minimise the peak sidelobe level

$$
\max _{u>0}\left|C_{A}(u)\right|
$$

by an integer greater than 1 (see [5] for a summary of known results). Another relaxation is to maximise the merit factor

$$
\frac{s^{2}}{2 \sum_{u>0}\left[C_{A}(u)\right]^{2}}
$$

(see [4] for a survey).

An alternative approach, which is considered here, is not to relax the Barker condition but to generalise $A$ from one to multiple dimensions. We define an $s_{1} \times \cdots \times s_{r}$ binary array to be an $r$-dimensional array $A=\left(a\left[i_{1}, \ldots, i_{r}\right]\right)$ whose elements satisfy

$$
a\left[i_{1}, \ldots, i_{r}\right]= \begin{cases}-1 \text { or } 1 & \text { if } 0 \leq i_{k}<s_{k} \text { for each } k=1, \ldots, r \\ 0 & \text { otherwise. }\end{cases}
$$

Each $s_{k}$ is assumed throughout to be an integer greater than 1, so that the array is "truly $r$-dimensional". The aperiodic autocorrelation function of an $s_{1} \times \cdots \times s_{r}$ binary array $A=\left(a\left[i_{1}, \ldots, i_{r}\right]\right)$ is given by

$$
C_{A}\left(u_{1}, \ldots, u_{r}\right):=\sum_{i_{1}} \ldots \sum_{i_{r}} a\left[i_{1}, \ldots, i_{r}\right] a\left[i_{1}+u_{1}, \ldots, i_{r}+u_{r}\right] \text { for integer } u_{1}, \ldots, u_{r} .
$$

An $s_{1} \times \cdots \times s_{r}$ Barker array is an $s_{1} \times \cdots \times s_{r}$ binary array for which

$$
\left|C_{A}\left(u_{1}, \ldots, u_{r}\right)\right| \leq 1 \text { for all }\left(u_{1}, \ldots, u_{r}\right) \neq(0, \ldots, 0) .
$$

Two-dimensional Barker arrays were introduced by Alquaddoomi and Scholtz [1] in 1989, who described their practical uses and exhibited the $2 \times 2$ example $\left[\begin{array}{cc}+ & + \\ + & -\end{array}\right]$. However they were unable to find any other sizes for a two-dimensional Barker array, and conjectured that no such size is possible. Their conjecture was recently proved: 
Theorem 1.3 (Davis, Jedwab and Smith 2006 [2]). There are no $s \times t$ Barker arrays except when $s=t=2$.

Multi-dimensional Barker arrays, of size $s_{1} \times \cdots \times s_{r}$ for $r>2$, were studied by Dymond [3]. She found several necessary conditions on the values $s_{k}$, by examining the existence question as three cases: none of the $s_{k}$ even; exactly one of the $s_{k}$ even; at least two of the $s_{k}$ even. The smallest sizes for which the existence of a Barker array is listed as undecided in [3] are $3 \times 3 \times 4,2 \times 3 \times 6$, and $2 \times 2 \times 3 \times 3$. Dymond's study of multi-dimensional Barker arrays concluded that:

Conjecture 1.4 (Dymond $1992[3]$ ). There are no $s_{1} \times \cdots \times s_{r}$ Barker arrays for $r>2$.

In this paper we give an elementary proof of Conjecture 1.4.

\section{Proof of Dymond's Conjecture}

We shall show that Conjecture 1.4 follows from the observation that a $(d+1)$-dimensional binary array can be mapped to a $d$-dimensional binary array, with the aperiodic autocorrelation functions of both arrays related in a simple way.

As an example of this mapping, let $A$ be the $3 \times 4$ binary array

$$
\left[\begin{array}{llll}
a_{00} & a_{01} & a_{02} & a_{03} \\
a_{10} & a_{11} & a_{12} & a_{13} \\
a_{20} & a_{21} & a_{22} & a_{23}
\end{array}\right]
$$

(writing $a_{i j}$ for $a[i, j]$ ), and concatenate the rows of $A$ to form the length 12 binary sequence

$$
B:=\left[\begin{array}{llllllllllll}
a_{00} & a_{01} & a_{02} & a_{03} & a_{10} & a_{11} & a_{12} & a_{13} & a_{20} & a_{21} & a_{22} & a_{23}
\end{array}\right] .
$$

It is easy to verify the equations

$$
C_{B}(-4 u)=C_{B}(4 u)=C_{A}(u, 0) \text { for } u=0,1 \text { or } 2
$$

and

$$
\left.\begin{array}{rl}
C_{B}(-v) & =C_{B}(v)=C_{A}(0, v)+C_{A}(1, v-4) \\
C_{B}(-4-v) & =C_{B}(4+v)=C_{A}(1, v)+C_{A}(2, v-4) \\
C_{B}(-8-v) & =C_{B}(8+v)=C_{A}(2, v)
\end{array}\right\} \text { for } v=1,2 \text { or } 3,
$$

which determine $C_{B}$ completely in terms of $C_{A}$. We can write these equations more concisely as

$$
C_{B}(4 u+v)=C_{A}(u, v)+C_{A}(u+1, v-4) \text { for integer } u, v, \text { where } 0 \leq v<4
$$

(noting that $C_{A}(u, v) \equiv C_{A}(-u,-v)$ for all integer $u, v$ ), so that each $C_{B}$ is the sum of exactly two terms $C_{A}$ (one or both of which might be trivially zero, according to the values of $u$ and $v$ ).

The following lemma formalises this observation and generalises it to the case where $A$ can have dimension greater than 2 . The proof is straightforward, although messy to write out, with the $3^{\text {rd }}$ and higher dimensions giving rise only to "dummy" indices. 
Lemma 2.1. Let $A=\left(a\left[i, j, i_{1}, \ldots, i_{r}\right]\right)$ be an $s \times t \times s_{1} \times \cdots \times s_{r}$ binary array (where the case $r=0$ is allowed). Define the st $\times s_{1} \times \cdots \times s_{r}$ binary array $B=\left(b\left[m, i_{1}, \ldots, i_{r}\right]\right)$ by $b\left[t i+j, i_{1}, \ldots, i_{r}\right]:=a\left[i, j, i_{1}, \ldots, i_{r}\right]$ for $0 \leq i<s, 0 \leq j<t, 0 \leq i_{k}<s_{k}(k=1, \ldots, r)$.

Then, for all integer $u, v, u_{1}, \ldots, u_{r}$, where $0 \leq v<t$,

$$
C_{B}\left(t u+v, u_{1}, \ldots, u_{r}\right)=C_{A}\left(u, v, u_{1}, \ldots, u_{r}\right)+C_{A}\left(u+1, v-t, u_{1}, \ldots, u_{r}\right) .
$$

Proof. Fix integers $u, v, u_{1}, \ldots, u_{r}$, where $0 \leq v<t$. From (1) we have

$$
C_{B}\left(t u+v, u_{1}, \ldots, u_{r}\right)=\sum_{m} \sum_{i_{1}} \ldots \sum_{i_{r}} b\left[m, i_{1}, \ldots, i_{r}\right] b\left[m+t u+v, i_{1}+u_{1}, \ldots, i_{r}+u_{r}\right] .
$$

Write each $m$ uniquely in the form $t i+j$, where $i, j$ are integers and $0 \leq j<t$, so that

$$
\begin{aligned}
C_{B}\left(t u+v, u_{1}, \ldots, u_{r}\right) \\
=\sum_{i} \sum_{j=0}^{t-1} \sum_{i_{1}} \ldots \sum_{i_{r}} b\left[t i+j, i_{1}, \ldots, i_{r}\right] b\left[t i+j+t u+v, i_{1}+u_{1}, \ldots, i_{r}+u_{r}\right] \\
=\sum_{i}\left(\sum_{j=0}^{t-v-1} \sum_{i_{1}} \ldots \sum_{i_{r}} b\left[t i+j, i_{1}, \ldots, i_{r}\right] b\left[t(i+u)+j+v, i_{1}+u_{1}, \ldots, i_{r}+u_{r}\right]\right. \\
\left.\quad+\sum_{j=t-v}^{t-1} \sum_{i_{1}} \ldots \sum_{i_{r}} b\left[t i+j, i_{1}, \ldots, i_{r}\right] b\left[t(i+u+1)+j+v-t, i_{1}+u_{1}, \ldots, i_{r}+u_{r}\right]\right) \\
=\sum_{i}\left(\sum_{j=0}^{t-v-1} \sum_{i_{1}} \ldots \sum_{i_{r}} a\left[i, j, i_{1}, \ldots, i_{r}\right] a\left[i+u, j+v, i_{1}+u_{1}, \ldots, i_{r}+u_{r}\right]\right. \\
\left.\quad+\sum_{j=t-v}^{t-1} \sum_{i_{1}} \ldots \sum_{i_{r}} a\left[i, j, i_{1}, \ldots, i_{r}\right] a\left[i+u+1, j+v-t, i_{1}+u_{1}, \ldots, i_{r}+u_{r}\right]\right)
\end{aligned}
$$

using (2). Then from (1) we have

$$
C_{B}\left(t u+v, u_{1}, \ldots, u_{r}\right)=C_{A}\left(u, v, u_{1}, \ldots, u_{r}\right)+C_{A}\left(u+1, v-t, u_{1}, \ldots, u_{r}\right),
$$

as required.

We now use Lemma 2.1 and a parity argument to show that in the case where $A$ is a Barker array, the array $B$ defined in (2) must also be a Barker array.

Theorem 2.2. If there exists an $s \times t \times s_{1} \times \cdots \times s_{r}$ Barker array (where the case $r=0$ is allowed) then there exists an st $\times s_{1} \times \cdots \times s_{r}$ Barker array. 
Proof. Let $A=\left(a\left[i, j, i_{1}, \ldots, i_{r}\right]\right)$ be an $s \times t \times s_{1} \times \cdots \times s_{r}$ Barker array, so that

$$
\left|C_{A}\left(u, v, u_{1}, \ldots u_{r}\right)\right| \leq 1 \text { for all }\left(u, v, u_{1}, \ldots u_{r}\right) \neq(0, \ldots, 0) .
$$

Define $B=\left(b\left[m, i_{1}, \ldots, i_{r}\right]\right)$ to be the $s t \times s_{1} \times \cdots \times s_{r}$ binary array given by $(2)$, and fix integers $u, v, u_{1}, \ldots, u_{r}$, where $0 \leq v<t$. By Lemma 2.1, $C_{B}\left(t u+v, u_{1}, \ldots, u_{r}\right)$ is the sum of two aperiodic autocorrelations of $A$, each of which is \pm 1 or 0 by (3). We claim that at least one of these autocorrelations of $A$ is zero, from which it follows that $B$ is a Barker array.

Suppose, for a contradiction, that $C_{A}\left(u, v, u_{1}, \ldots, u_{r}\right)$ and $C_{A}\left(u+1, v-t, u_{1}, \ldots, u_{r}\right)$ are both nonzero. Then by $(1), C_{A}\left(u, v, u_{1}, \ldots, u_{r}\right)$ is the sum of exactly $(s-|u|)(t-$ $|v|) \prod_{k}\left(s_{k}-\left|u_{k}\right|\right)$ terms, each of which is \pm 1 , so that

$$
C_{A}\left(u, v, u_{1}, \ldots, u_{r}\right) \equiv(s+u)(t+v) \prod_{k}\left(s_{k}+u_{k}\right) \quad(\bmod 2) .
$$

Since $C_{A}\left(u, v, u_{1}, \ldots, u_{r}\right) \neq 0$ by assumption, (3) then implies that

$$
(s+u)(t+v) \prod_{k}\left(s_{k}+u_{k}\right) \equiv 1 \quad(\bmod 2)
$$

and so $(s+u)$ is odd. But a similar argument applied to $C_{A}\left(u+1, v-t, u_{1}, \ldots, u_{r}\right)$ shows that

$$
(s+u+1) v \prod_{k}\left(s_{k}+u_{k}\right) \equiv 1 \quad(\bmod 2),
$$

and so $(s+u+1)$ is odd. This gives the required contradiction.

Conjecture 1.4 now follows directly.

Corollary 2.3. There are no $s_{1} \times \cdots \times s_{r}$ Barker arrays for $r>2$.

Proof. Suppose, for a contradiction, that there exists an $s_{1} \times \cdots \times s_{r}$ Barker array with $r>2$ (where $s_{k} \geq 2$ for each $k=1, \ldots, r$ ). Apply Theorem 2.2 repeatedly to produce an $s \times t$ Barker array having $s t \geq 2^{3}$. This contradicts Theorem 1.3.

The case $r=0$ of Theorem 2.2, namely that the existence of an $s \times t$ Barker array implies the existence of a Barker sequence of length st, is of special interest. In particular we can interpret the existence of a Barker sequence of length 4 as being a consequence of the existence of a $2 \times 2$ Barker array.

If the converse of the case $r=0$ of Theorem 2.2 were to hold (so that the existence of a Barker sequence of length st with $s, t>1$ implies the existence of an $s \times t$ Barker array) then it would imply Conjecture 1.1, as follows. Suppose, for a contradiction, that a Barker sequence of length $s>13$ exists. By Theorem 1.2, we can write $s=2 t$ for some integer $t>1$. By the hypothetical converse, a Barker array of size $2 \times t$ then exists and so by Theorem 1.3, $t=2$. The Barker sequence therefore has length $s=2 t=4$, which contradicts the assumption that $s>13$ and proves Conjecture 1.1. However we do not know what strategy to use to attempt to prove this hypothetical converse. One possibility might be to identify a mapping from a $d$-dimensional array to a $(d+1)$-dimensional array that somehow acts as a converse to Lemma 2.1. 


\section{Acknowledgement}

The authors are grateful to the referees for their helpful comments.

\section{References}

[1] S. Alquaddoomi and R.A. Scholtz. On the nonexistence of Barker arrays and related matters. IEEE Trans. Inform. Theory, 35:1048-1057, 1989.

[2] J.A. Davis, J. Jedwab, and K.W. Smith. Proof of the Barker array conjecture. Proc. Amer. Math. Soc., 2006. To appear.

[3] M. Dymond. Barker arrays: existence, generalization and alternatives. PhD thesis, University of London, 1992.

[4] J. Jedwab. A survey of the merit factor problem for binary sequences. In T. Helleseth et al., editors, Sequences and Their Applications - Proceedings of SETA 2004, volume 3486 of Lecture Notes in Computer Science, pages 30-55. Springer-Verlag, Berlin Heidelberg, 2005.

[5] J. Jedwab and K. Yoshida. The peak sidelobe level of families of binary sequences. IEEE Trans. Inform. Theory, 52:2247-2254, 2006.

[6] K.H. Leung and B. Schmidt. The field descent method. Designs, Codes and Cryptography, 36:171-188, 2005.

[7] B. Schmidt. Characters and Cyclotomic Fields in Finite Geometry, volume 1797 of Lecture Notes in Mathematics. Springer, Berlin, 2002.

[8] R. Turyn and J. Storer. On binary sequences. Proc. Amer. Math. Soc., 12:394-399, 1961.

[9] R.J. Turyn. On Barker codes of even length. Proceedings of the IEEE, 51:1256, 1963.

[10] R.J. Turyn. Character sums and difference sets. Pacific J. Math., 15:319-346, 1965.

[11] R.J. Turyn. Sequences with small correlation. In H.B. Mann, editor, Error Correcting Codes, pages 195-228. Wiley, New York, 1968. 